\title{
CONCENTRATION FUNCTION FOR PYRAMID AND QUANTUM METRIC MEASURE SPACE
}

\author{
RYUNOSUKE OZAWA
}

(Communicated by Thomas Schlumprecht)

\begin{abstract}
In this paper, we generalize the concentration function for metric measure space to one for pyramid and quantum metric measure space. We also study the limit of the concentration function for convergent sequences of pyramids and quantum metric measure spaces.
\end{abstract}

\section{INTRODUCTION}

Gromov [7, Chapter $3 \cdot \frac{1}{2}_{+}$] introduced the box distance function $\square$ and the observable distance function $d_{\text {conc }}$ on the set $\mathcal{X}$ of isomorphism classes of mm-spaces (metric measure spaces). The box distance function is a simple and natural distance function on $\mathcal{X}$. Vershik suggested constructing a compactification of $\left(\mathcal{X}_{1}, \square\right)$ and defining basic metric measure invariants for an element of compactification, where we denote by $\mathcal{X}_{1}$ the set of isomorphism classes of mm-spaces with diameter at most one (see [17] and [7, Section 3. $\left.\frac{1}{2} \cdot 7\right]$ ). An answer is given by Elek [2]. He introduced a qmm-space (quantum metric measure space) and proved that the set of isomorphism classes of qmm-spaces $\mathcal{Q}_{1}$ is a compactification of $\left(\mathcal{X}_{1}, \square\right)$. The idea of qmm-space comes from the graph limit theory due to Lovász-Szegedy [12]. He also extended two metric measure invariants called the observable diameter and the separation distance to qmm-spaces and proved some limit formulas for a convergent sequence in $\mathcal{Q}_{1}$.

The observable distance function comes from the idea of concentration of measure phenomenon due to Lévy and Milman. Gromov [7, Chapter 3. $\frac{1}{2}{ }_{+}$] introduced a pyramid and proved that the set of pyramids $\Pi$ is a compactification of $\left(\mathcal{X}, d_{\text {conc }}\right)$. Moreover, Shioya [15, 16] constructed a metric on $\Pi$ which is compatible with the topology of the compactification. This compactification is useful to describe the asymptotic behavior of a sequence of Riemannian manifolds with unbounded dimension (see [13 16]). Ozawa-Shioya [13] extended the observable diameter and the separation distance to $\Pi$ and proved some limit formulas for a convergent sequence in $\Pi$, which are applied to study a significant property of the asymptotic behavior of a sequence of pyramids, so-called the phase transition property.

The concentration function is one of the most important invariants of an mmspace as well as the observable diameter and the separation distance are.

Received by the editors October 17, 2015 and, in revised form, May 5, 2016.

2010 Mathematics Subject Classification. Primary 53C23, 28Axx.

Key words and phrases. Metric measure space, concentration function, observable distance, box distance, pyramid, quantum metric measure space.

This work was supported by Research Fellowships of the Japan Society for the Promotion of Science for Young Scientists (Grant Number 13J02664). 
This was introduced by Amir-Milman [1] and is related to the concentration of measure phenomenon. It is a natural problem to investigate the limit of concentration functions for a convergent sequence of mm-spaces. In this paper, we generalize the concentration function for an element of $\Pi$ and $\mathcal{Q}_{1}$, and prove limit formulas for a convergent sequence in $\Pi$ and $\mathcal{Q}_{1}$. Denote by $\alpha_{\mathcal{P}}(r, \kappa)$ and $\alpha_{Q}(r, \kappa)$ the concentration function of a pyramid $\mathcal{P}$ and a qmm-space $Q$ (see Definitions 3.3 and 4.1). Convergences in $\Pi$ and $\mathcal{Q}_{1}$ are called weak convergence and convergence in sampling respectively.

Our main theorems are stated as follows.

Theorem 1.1 (Limit formula). Let $\mathcal{P}$ and $\mathcal{P}_{n}, n=1,2, \ldots$, be pyramids. If $\mathcal{P}_{n}$ converges weakly to $\mathcal{P}$ as $n \rightarrow \infty$, then

$$
\begin{aligned}
\alpha_{\mathcal{P}}(r, \kappa) & =\lim _{\delta \rightarrow 0+} \liminf _{n \rightarrow \infty} \alpha_{\mathcal{P}_{n}}(r-\delta, \kappa-\delta) \\
& =\lim _{\delta \rightarrow 0+} \limsup _{n \rightarrow \infty} \alpha_{\mathcal{P}_{n}}(r-\delta, \kappa-\delta)
\end{aligned}
$$

for any $r>0$ and $0<\kappa \leq 1$.

Theorem 1.2 (Limit inequality). Let $Q$ and $Q_{n}, n=1,2, \ldots$, be qmm-spaces. If $Q_{n}$ converges to $Q$ in sampling as $n \rightarrow \infty$, then

$$
\lim _{\delta \rightarrow 0+} \limsup _{n \rightarrow \infty} \alpha_{Q_{n}}(r-\delta, \kappa-\delta) \leq \alpha_{Q}(r, \kappa)
$$

for any $0<r, \kappa \leq 1$.

Note that if $Q$ and $Q_{n}, n=1,2, \ldots$, are mm-spaces, we have the equation in Theorem 1.2 (see Remark 4.11). We do not have the reverse inequality in general. One of the counterexamples for the reverse inequality is the sequence $\left\{S^{n}\left(\pi^{-1}\right)\right\}_{n=1}^{\infty}$ of $n$-dimensional spheres equipped with the geodesic distance and radius $\pi^{-1}$ (see Proposition 4.12 and Remark 4.13).

\section{Preliminaries}

In this section, we give the definitions and compactification theorems stated in [7. Chapter $3 \frac{1}{2}{ }_{+}$], [10, [5], [15], [16], and [2].

\section{1. mm-Isomorphism, Lipschitz order, and distance matrix distribution.}

Definition 2.1 (mm-Space). A triple $X=\left(X, d_{X}, \mu_{X}\right)$ is called an mm-space (metric measure space) if $\left(X, d_{X}\right)$ is a complete separable metric space and if $\mu_{X}$ is a Borel probability measure on $X$.

Definition 2.2 (mm-Isomorphism). Two mm-spaces $X$ and $X^{\prime}$ are said to be $m m$ isomorphic to each other if there exists an isometry $f: \operatorname{supp}\left(\mu_{X}\right) \rightarrow \operatorname{supp}\left(\mu_{X^{\prime}}\right)$ such that $f_{*} \mu_{X}=\mu_{X^{\prime}}$, where $f_{*} \mu_{X}$ is the push-foward measure of $\mu_{X}$ by $f$. Such an $f$ is called an mm-isomorphism.

Note that $X$ is mm-isomorphic to $\left(\operatorname{supp}\left(\mu_{X}\right), d_{X}, \mu_{X}\right)$. Denote by $\mathcal{X}\left(\right.$ resp. $\left.\mathcal{X}_{1}\right)$ the set of mm-isomorphism classes of mm-spaces (resp. the set of mm-isomorphism classes of mm-spaces with diameter at most one).

Definition 2.3 (Lipschitz order). Let $X$ and $X^{\prime}$ be two mm-spaces. We say that $X$ (Lipschitz) dominates $X^{\prime}$ and write $X^{\prime} \prec X$ if there exists a 1-Lipschitz map $f: X \rightarrow X^{\prime}$ with $f_{*} \mu_{X}=\mu_{X^{\prime}}$. We call the relation $\prec$ on $\mathcal{X}$ the Lipschitz order. 
The Lipschitz order $\prec$ is a partial order relation on $\mathcal{X}$.

Let $X$ be a metric space. Denote by $M_{N}(X)$ the set of all $X$-valued symmetric matrices of order $N$ equipped with $l_{\infty}$-product metric, and by $M_{\infty}(X)$ the set of all $X$-valued symmetric matrices of infinite-order. We equipped $M_{\infty}(X)$ with the coarsest topology such that the natural projection $\pi_{N}^{X}: M_{\infty}(X) \rightarrow M_{N}(X)$ defined by $\pi_{N}^{X}\left(\left(x_{i, j}\right)_{i, j=1}^{\infty}\right):=\left(x_{i, j}\right)_{i, j=1}^{N}$ is continuous for any natural number $N$. We write $\pi_{N}$ omitting $X$ whenever there is no confusion. Let $T$ be a topological space. Denote by $\mathcal{B}(T)$ the set of Borel sets on $T$, and by $\mathcal{M}(T)$ the set of Borel probability measures on $T$ equipped with the weak topology.

Definition 2.4 (Distance matrix distribution). Let $X$ be an mm-space and $N \in$ $\mathbb{N} \cup\{\infty\}$. Define a map $K_{N}^{X}: X^{N} \rightarrow M_{N}(\mathbb{R})$ by

$$
K_{N}^{X}\left(x_{1}, \ldots, x_{N}\right):=\left(d_{X}\left(x_{i}, x_{j}\right)\right)_{i, j=1}^{N},
$$

and the $N$-dimensional distance matrix distribution $\underline{\mu}_{N}^{X}$ of $X$ by

$$
\underline{\mu}_{N}^{X}:=\left(K_{N}^{X}\right)_{*} \mu_{X}^{\otimes N} .
$$

The $N$-dimensional distance matrix distribution is a Borel probability measure on $M_{N}(\mathbb{R})$. Define the map $\tau: \mathcal{X} \rightarrow \mathcal{M}\left(M_{\infty}(\mathbb{R})\right)$ by $\tau(X):=\underline{\mu}_{\infty}^{X}$.

Theorem 2.5 (mm-Reconstruction theorem, 17, Section 2, Theorem], 7, Section $\left.3 \frac{1}{2} .5,3 \frac{1}{2} .7\right]$, [9, Theorem 2.1], [15, Theorem 4.7]). Let $X$ and $X^{\prime}$ be two mm-spaces. The following (1), (2), and (3) are equivalent to each other:

(1) $X$ and $X^{\prime}$ are isomorphic to each other.

(2) $\underline{\mu}_{N}^{X}=\underline{\mu}_{N}^{X^{\prime}}$ for all $N \in \mathbb{N}$.

(3) $\underline{\mu}_{\infty}^{X}=\underline{\mu}_{\infty}^{X^{\prime}}$.

This theorem means that the infinite-dimensional distance matrix distribution is a complete invariant of $\mathrm{mm}$-spaces.

Lemma 2.6 ([9. Lemma 2.2]). Let $X$ be an mm-space and $N \in \mathbb{N}$. Then we have $\left(\pi_{N}\right)_{*} \underline{\mu}_{\infty}^{X}=\underline{\mu}_{N}^{X}$

2.2. Concentration function. The concentration function is one of the most fundamental invariants of an mm-space.

For a subset $A$ of a metric space $\left(X, d_{X}\right)$ and for a real number $r>0$, we set

$$
U_{r}(A):=\left\{x \in X \mid d_{X}(x, A)<r\right\},
$$

where $d_{X}(x, A):=\inf _{a \in A} d_{X}(x, a)$.

Definition 2.7 (Concentration function of mm-space). Let $r>0$ and $0<\kappa \leq 1$. The concentration function of $m m$-space $X$ is defined to be

$$
\alpha_{X}(r, \kappa):=\sup \left\{1-\mu_{X}\left(U_{r}(A)\right) \mid A \in \mathcal{B}(X), \mu_{X}(A) \geq \kappa\right\} .
$$

Lemma 2.8 ([10, Lemma 1.1]). Let $X$ be an $m m$-space and $0<\kappa \leq 1$. Then we have

$$
\alpha_{X}\left(r+r_{0}, \kappa\right) \leq \alpha_{X}(r, 1 / 2)
$$

for any $r>0$ and $r_{0}>0$ satisfying $\alpha_{X}\left(r_{0}, 1 / 2\right)<\kappa$.

Definition 2.9 (Lévy family). A sequence of mm-spaces $X_{n}, n=1,2, \ldots$, is called a Lévy family if for any $r>0$ and $0<\kappa \leq 1, \alpha_{X_{n}}(r, \kappa)$ converges to zero as $n \rightarrow \infty$. 


\subsection{Box distance and observable distance.}

Definition 2.10 (Prokhorov metric). Let $X$ be a metric space. For two Borel probability measures $\mu$ and $\nu$ on $X$, we define the Prokhorov distance $d_{\operatorname{Pr}}(\mu, \nu)$ between $\mu$ and $\nu$ by

$$
d_{\operatorname{Pr}}(\mu, \nu):=\inf \left\{\varepsilon>0 \mid \mu(A) \leq \nu\left(U_{\varepsilon}(A)\right)+\varepsilon \text { for any Borel set } A \subset X\right\} .
$$

The distance function $d_{\mathrm{Pr}}$ is called the Prokhorov metric on the set of probability measures on $X$.

The Prokhorov metric is a metrization of weak convergence of Borel probability measures on $X$ provided that $X$ is a separable metric space.

Definition 2.11 (Ky Fan metric). Let $(T, \mu)$ be a measure space and $X$ be a metric space. For two $\mu$-measurable maps $f, g: T \rightarrow X$, we define the $K y$ Fan distance between $f$ and $g$ by

$$
d_{\mathrm{KF}}^{\mu}(f, g):=\inf \left\{\varepsilon>0 \mid \mu\left(\left\{t \in T \mid d_{X}(f(t), g(t))>\varepsilon\right\}\right) \leq \varepsilon\right\} .
$$

The distance function $d_{\mathrm{KF}}^{\mu}$ is called the Ky Fan metric on the set of $\mu$-measurable maps from $T$ to $X$.

The Ky Fan metric is a metrization of convergence in measure.

Definition 2.12 (Parameter). Let $I:=[0,1]$ and $T=\left(T, \mu_{T}\right)$ be a Polish topological space equipped with a Borel probability measure. A Borel measurable map $\varphi: I \rightarrow T$ is called a parameter of $T$ if $\varphi$ satisfies the following:

(1) $\varphi_{*} \mathcal{L}=\mu_{T}$, where $\mathcal{L}$ is the Lebesgue measure on $I$.

(2) The image of any Borel set is a Borel set of $\varphi(I)$.

The definition of a parameter is not usual. The usual definition of a parameter is only by (1). We put (2) as an additional condition for measure theoretic approach. Any Polish topological space equipped with a Borel probability measure has a parameter.

Definition 2.13 (Box distance between two mm-spaces). We define the box distance $\square\left(X, X^{\prime}\right)$ between two mm-spaces $X$ and $X^{\prime}$ to be the infimum of $\varepsilon \geq 0$ such that there exist parameters $\varphi: I \rightarrow X, \psi: I \rightarrow X^{\prime}$, and Borel subset $I_{0} \subset I$ such that

$$
\begin{aligned}
& \left|\varphi^{*} d_{X}(s, t)-\psi^{*} d_{X^{\prime}}(s, t)\right| \leq \varepsilon \quad \text { for any } s, t \in I_{0} ; \\
& \mathcal{L}\left(I_{0}\right) \geq 1-\varepsilon,
\end{aligned}
$$

where $\varphi^{*} d_{X}(s, t):=d_{X}(\varphi(s), \varphi(t))$ for $s, t \in I$.

The box distance function $\square$ is a metric on $\mathcal{X}$.

Combining [6. Theorem 5] and [11, Theorem 3.1], we have the next theorem. Note that the proof of the next theorem is omitted in the original article (see [7. Section $\left.\left.3 \cdot \frac{1}{2} \cdot 14\right]\right)$.

Theorem 2.14. Let $\left\{X_{n}\right\}_{n=1}^{\infty}$ be a sequence of mm-spaces and let $X$ be an mmspace. The following (1), (2), and (3) are equivalent to each other:

(1) $X_{n} \square$-converges to $X$.

(2) $\underline{\mu}_{N}^{X_{n}}$ converges weakly to $\underline{\mu}_{N}^{X}$ for all $N \in \mathbb{N}$.

(3) $\underline{\mu}_{\infty}^{X_{n}}$ converges weakly to $\underline{\mu}_{\infty}^{X}$. 
Definition 2.15 (Observable distance). Denote by $\mathcal{L} i p_{1}(X)$ the set of 1-Lipschitz continuous functions on an mm-space $X$. For any parameter $\varphi$ of $X$, we set

$$
\varphi^{*} \mathcal{L} i p_{1}(X):=\left\{f \circ \varphi \mid f \in \mathcal{L} i p_{1}(X)\right\} .
$$

We define the observable distance $d_{\text {conc }}\left(X, X^{\prime}\right)$ between two $m m$-spaces $X$ and $X^{\prime}$ by

$$
d_{\text {conc }}\left(X, X^{\prime}\right):=\inf _{\varphi, \psi} d_{\mathrm{H}}\left(\varphi^{*} \mathcal{L} i p_{1}(X), \psi^{*} \mathcal{L} i p_{1}\left(X^{\prime}\right)\right),
$$

where $\varphi: I \rightarrow X$ and $\psi: I \rightarrow X^{\prime}$ run over all parameters of $X$ and $X^{\prime}$, respectively, and where $d_{\mathrm{H}}$ is the Hausdorff distance function with respect to the Ky Fan metric $d_{\mathrm{KF}}^{\mathcal{L}}$.

The observable distance $d_{\text {conc }}$ is a metric on $\mathcal{X}$. Note that $d_{\text {conc }}\left(X, X^{\prime}\right) \leq$ $\square\left(X, X^{\prime}\right)$ for any two mm-spaces $X$ and $X^{\prime}$.

Proposition 2.16 ([7, Section $\left.3 \frac{1}{2} .36\right]$ ). Let $\left\{X_{n}\right\}_{n=1}^{\infty}$ be a sequence of mm-spaces. Then $\left\{X_{n}\right\}_{n=1}^{\infty}$ is a Lévy family if and only if $X_{n} d_{\text {conc-converges to a one-point }}$ $m m$-space as $n \rightarrow \infty$.

\subsection{Pyramid.}

Definition 2.17 (Pyramid). A subset $\mathcal{P} \subset \mathcal{X}$ is called a pyramid if it satisfies the following conditions:

(1) If $X \in \mathcal{P}$ and if $X^{\prime} \prec X$, then $X^{\prime} \in \mathcal{P}$.

(2) For any $X, X^{\prime} \in \mathcal{P}$, there exists $Z \in \mathcal{P}$ such that $X \prec Z$ and $X^{\prime} \prec Z$.

(3) $\mathcal{P}$ is a non-empty $\square$-closed set.

We denote the set of pyramids by $\Pi$.

For an mm-space $X$, we define

$$
\mathcal{P}_{X}:=\left\{X^{\prime} \in \mathcal{X} \mid X^{\prime} \prec X\right\} .
$$

Then $\mathcal{P}_{X}$ is a pyramid.

In Gromov's book [7, Section 3. $\frac{1}{2} .51$ ], the definition of a pyramid is only by (1) and (2) of Definition 2.17. Shioya put (3) as an additional condition for the Hausdorff property of $\Pi$ (see Theorem 2.20).

Definition $2.18((N, R)$-measurement). Let $\mathcal{P}$ be a pyramid, $N$ a natural number, and $R$ a non-negative real number. Denote by $B_{R}^{N}:=\left\{x \in \mathbb{R}^{N} \mid\|x\|_{\infty} \leq R\right\}$. We define

$$
\mathcal{M}(\mathcal{P} ; N, R):=\left\{\mu \in \mathcal{M}\left(B_{R}^{N}\right) \mid\left(B_{R}^{N},\|\cdot\|_{\infty}, \mu\right) \in \mathcal{P}\right\} .
$$

We call $\mathcal{M}(\mathcal{P} ; N, R)$ the $(N, R)$-measurement of $\mathcal{P}$.

The $(N, R)$-measurement $\mathcal{M}(\mathcal{P} ; N, R)$ is a compact subset of $\mathcal{M}\left(\mathbb{R}^{N}\right)$.

Definition 2.19. For two pyramids $\mathcal{P}, \mathcal{P}^{\prime}$, and for a positive real number $R$, we define

$$
\rho_{R}\left(\mathcal{P}, \mathcal{P}^{\prime}\right):=\sum_{N=1}^{\infty} \frac{1}{N 2^{N+1}} d_{\mathrm{H}}\left(\mathcal{M}(\mathcal{P} ; N, N R), \mathcal{M}\left(\mathcal{P}^{\prime} ; N, N R\right)\right),
$$

where $d_{\mathrm{H}}$ is the Hausdorff distance function with respect to the Prokhorov metric $d_{\mathrm{Pr}}$. 
Theorem 2.20 ([7, Section 3. $\left.\frac{1}{2} .55\right]$, [15, Theorem 7.27], [16, Theorem 1.2, Proposition 3.5], [13, Theorem 3.7]). We have the following:

(1) $\rho_{R}$ for each $R>0$ is a metric on $\Pi$. Moreover, $\left(\Pi, \rho_{R}\right)$ for all $R>0$ are homeomorphic to each other.

(2) The metric space $\left(\Pi, \rho_{R}\right)$ is compact.

(3) The map $\mathcal{X} \ni X \mapsto \mathcal{P}_{X} \in \Pi$ is a topological embedding with respect to $d_{\text {conc }}$ and $\rho_{R}$, and its image is dense on $\Pi$. In particular, $\left(\Pi, \rho_{R}\right)$ is a compactfication of $\left(\mathcal{X}, d_{\text {conc }}\right)$.

(4) For any two pyramids $\mathcal{P}, \mathcal{P}^{\prime}$, for any natural number $N$, and for any positive real number $R$, we have

$$
d_{\mathrm{H}}\left(\mathcal{M}(\mathcal{P} ; N, N R), \mathcal{M}\left(\mathcal{P}^{\prime} ; N, N R\right)\right) \leq N 2^{N+1} \rho_{R}\left(\mathcal{P}, \mathcal{P}^{\prime}\right) .
$$

We say that a sequence of pyramids $\mathcal{P}_{n}, n=1,2, \ldots$, converges weakly to a pyramid $\mathcal{P}$ if $\mathcal{P}_{n} \rho_{R}$-converges to $\mathcal{P}$ as $n \rightarrow \infty$.

\subsection{Quantum metric measure space.}

Definition 2.21 (qmm-Space). A triple $Q=\left(Q, \mu_{Q}, d_{Q}^{*}\right)$ is called a qmm-space (quantum metric measure space) if it satisfies the following:

(1) $\left(Q, \mu_{Q}\right)$ is a Polish topological space with a Borel probability measure.

(2) A measurable map $d_{Q}^{*}: Q \times Q \rightarrow \mathcal{M}(I)$ satisfies $d_{Q}^{*}(q, q)=\delta_{0}$ a.s. $q \in Q$ and $d_{Q}^{*}\left(q, q^{\prime}\right)=d_{Q}^{*}\left(q^{\prime}, q\right)$ a.s. $\left(q, q^{\prime}\right) \in Q^{2}$.

(3) For any $t_{i, j} \in \operatorname{supp}\left(d_{Q}^{*}\left(q_{i}, q_{j}\right)\right), i, j=1,2,3$, we have

$$
t_{1,3} \leq t_{1,2}+t_{2,3}
$$

a.s. $\left(q_{1}, q_{2}, q_{3}\right) \in Q^{3}$.

For any mm-space $X$ with diameter at most one, we define

$$
d_{X}^{*}\left(x_{1}, x_{2}\right):=\delta_{d_{X}\left(x_{1}, x_{2}\right)} .
$$

Then

$$
Q_{X}:=\left(X, \mu_{X}, d_{X}^{*}\right)
$$

is a qmm-space. Note that any qmm-space has a parameter.

Definition 2.22 (Box distance between two qmm-spaces). We define the box distance $\square_{\mathcal{Q}}\left(Q, Q^{\prime}\right)$ between two qmm-spaces $Q$ and $Q^{\prime}$ to be the infimum of $\varepsilon \geq 0$ such that there exist parameters $\varphi: I \rightarrow Q, \psi: I \rightarrow Q^{\prime}$, and Borel subset $I_{0} \subset I$ such that

$$
\begin{aligned}
& d_{\operatorname{Pr}}\left(\varphi^{*} d_{Q}^{*}(s, t), \psi^{*} d_{Q^{\prime}}^{*}(s, t)\right) \leq \varepsilon \quad \text { for any } s, t \in I_{0} \\
& \mathcal{L}\left(I_{0}\right) \geq 1-\varepsilon,
\end{aligned}
$$

where $\varphi^{*} d_{Q}^{*}(s, t):=d_{Q}^{*}(\varphi(s), \varphi(t))$ for $s, t \in I$.

For any $X, X^{\prime} \in \mathcal{X}_{1}$, we have $\square_{\mathcal{Q}}\left(Q_{X}, Q_{X^{\prime}}\right)=\square\left(X, X^{\prime}\right)$. For any Borel probability measure $\mu$ on $I$, denote by $\downarrow \mu:=\min \{t \mid t \in \operatorname{supp}(\mu)\}$ and $\uparrow \mu:=\max \{t \mid t \in$ $\operatorname{supp}(\mu)\}$.

Remark 2.23. By the definition of qmm-space, we have $\uparrow d_{Q}^{*}\left(q, q^{\prime}\right)=\downarrow d_{Q}^{*}\left(q, q^{\prime}\right)$ a.s. $\left(q, q^{\prime}\right) \in Q^{2}$.

Definition 2.24 (qmm-Isomorphism). Two qmm-spaces $Q$ and $Q^{\prime}$ are said to be qmm-isomorphic to each other if $\square_{\mathcal{Q}}\left(Q, Q^{\prime}\right)=0$. 
Denote by $\mathcal{Q}_{1}$ the set of isomorphism classes of qmm-space.

Remark 2.25. (1) The box distance function $\square_{\mathcal{Q}}$ is a metric on $\mathcal{Q}_{1}$.

(2) For any parameter $p$ of $Q,\left(Q, \mu_{Q}, d_{Q}^{*}\right)$ and $\left(I, \mathcal{L}^{1}, p^{*} d_{Q}^{*}\right)$ are qmm-isomorphic to each other.

Definition 2.26 (Quantum distance matrix distribution). Let $Q$ be a qmm-space. Define a map $K_{\infty}^{Q}: Q^{\infty} \rightarrow M_{\infty}(\mathcal{M}(I))$ by

$$
K_{\infty}^{Q}\left(\left\{q_{n}\right\}_{n=1}^{\infty}\right):=\left(d_{Q}^{*}\left(q_{i}, q_{j}\right)\right)_{i, j=1}^{\infty},
$$

and the infinite-dimensional quantum distance matrix distribution $\underline{\nu}_{\infty}^{Q}$ of $Q$ by

$$
\underline{\nu}_{\infty}^{Q}:=\left(K_{\infty}^{Q}\right)_{*} \mu_{Q}^{\otimes \infty}
$$

The infinite-dimensional quantum distance matrix distribution is a Borel probability measure on $M_{\infty}(\mathcal{M}(I))$. Consider it as a Borel probability measure on $\mathcal{M}\left(M_{\infty}(I)\right)$.

Definition 2.27 (Barycenter). Let $E$ be a Banach space and $E^{*}$ be the dual space with the weak topology. We give a compact convex subset $C \subset E^{*}$ and a Borel probability measure $\mu$ on $C$. A point $b \in C$ is called a barycenter of $\mu$ if

$$
\langle b, v\rangle=\int_{C}\langle f, v\rangle d \mu(f)
$$

for any $v \in E$, where $\langle f, v\rangle$ is a dual coupling of $f \in C$ and $v \in E$.

There is a unique barycenter for any Borel probability measure $\mu$ on a compact convex subset $C$. We set $E=C_{b}\left(M_{\infty}(I)\right)$ the set of bounded continuous functions on $M_{\infty}(I), E^{*}$ the set of Radon measures on $M_{\infty}(I), C=\mathcal{M}\left(M_{\infty}(I)\right)$, and $\mu=\underline{\nu}_{\infty}^{Q}$ as in Definition 2.27 and for a qmm-space $Q$. Denote by $\underline{b}_{\infty}^{Q}$ the barycenter of $\underline{\nu}_{\infty}^{Q}$. We have $\underline{b}_{\infty}^{Q_{X}}=\underline{\mu}_{\infty}^{X}$ for any $X \in \mathcal{X}_{1}$.

Theorem 2.28 ([2], Theorem 2]). Let $Q$ and $Q^{\prime}$ be two qmm-spaces. Then $\square_{\mathcal{Q}}\left(Q, Q^{\prime}\right)=0$ if and only if $\underline{b}_{\infty}^{Q}=\underline{b}_{\infty}^{Q^{\prime}}$. Moreover if $\square_{\mathcal{Q}}\left(Q, Q^{\prime}\right)=0$, there exist parameters $\varphi: I \rightarrow Q$ and $\psi: I \rightarrow Q^{\prime}$ such that $\varphi^{*} d_{Q}^{*}(s, t)=\psi^{*} d_{Q^{\prime}}^{*}(s, t)$ for a.s. $(s, t) \in I^{2}$.

Since $\mathcal{M}\left(M_{\infty}(I)\right)$ is a compact metric space, so is the closure $\overline{\tau\left(\mathcal{X}_{1}\right)}$.

Theorem 2.29 ([2, Theorem 1, Section 5]). We have the following:

(1) For any qmm-space $Q$, its barycenter $\underline{b}_{\infty}^{Q}$ is an element of the closure $\overline{\tau\left(\mathcal{X}_{1}\right)}$.

(2) For any $\mu \in \overline{\tau\left(\mathcal{X}_{1}\right)}$, there exists a qmm-space $Q$ such that $\mu=\underline{b}_{\infty}^{Q}$.

In particular, $\mathcal{Q}_{1}$ is a compactification of $\left(\mathcal{X}_{1}, \square\right)$.

We say that a sequence of qmm-spaces $Q_{n}, n=1,2, \ldots$, converges to a qmmspace $Q$ in sampling if $\underline{b}_{\infty}^{Q_{n}}$ converges weakly to $\underline{b}_{\infty}^{Q}$.

Remark 2.30. Since the sequence $\left\{S^{n}\left(\pi^{-1}\right)\right\}_{n=1}^{\infty}$ of $n$-dimensional sphere equipped with geodesic distance and radius $\pi^{-1}$ does not have a Cauchy subsequence with respect to the box distance function (see [4, Proposition 3.1]), we obtain that $\left(\mathcal{Q}_{1}, \square_{\mathcal{Q}}\right.$ ) is not a compact metric space. The topology generated by the box distance function on $\mathcal{Q}_{1}$ is not compatible with the topology of the convergence in sampling. 


\section{Concentration FunCtion FOR A PyRAmid}

Lemma 3.1. Let $X$ be an mm-space. Then we have

$$
\alpha_{X}(r, \kappa)=\lim _{\delta, \delta^{\prime} \rightarrow 0+} \alpha_{X}\left(r-\delta, \kappa-\delta^{\prime}\right)
$$

for any $r>0$ and $0<\kappa \leq 1$.

Proof. Let $\left\{\delta_{n}\right\}_{n=1}^{\infty}$ and $\left\{\delta_{n}^{\prime}\right\}_{n=1}^{\infty}$ be monotone decreasing sequences of positive real numbers converging to zero. Then, $\alpha_{X}\left(r-\delta_{n}, \kappa-\delta_{n}^{\prime}\right)$ is monotone non-increasing in $n$. We set

$$
\beta:=\lim _{n \rightarrow \infty} \alpha_{X}\left(r-\delta_{n}, \kappa-\delta_{n}^{\prime}\right) .
$$

Since $\alpha_{X}\left(r-\delta_{n}, \kappa-\delta_{n}^{\prime}\right) \geq \alpha_{X}(r, \kappa)$, we have $\beta \geq \alpha_{X}(r, \kappa)$. It suffices to prove $\alpha_{X}(r, \kappa) \geq \beta$. It follows from the definition of $\beta$ that there exist Borel subsets $A_{n} \subset X$ such that $\mu_{X}\left(A_{n}\right) \geq \kappa-\delta_{n}^{\prime}$ for any $n \in \mathbb{N}$ and

$$
1-\lim _{n \rightarrow \infty} \mu_{X}\left(U_{r-\delta_{n}}\left(A_{n}\right)\right)=\beta .
$$

We may assume that $A_{n}$ is a closed set. Take a monotone decreasing sequence $\left\{\eta_{p}\right\}_{p=1}^{\infty}$ of positive real numbers converging to zero. The inner regularity of $\mu_{X}$ proves that there are compact subsets $\left\{K_{p}\right\}_{p=1}^{\infty}$ such that $\mu_{X}\left(K_{p}\right)>1-\eta_{p}$ and $K_{p} \subset K_{p+1}$ for any $p \in \mathbb{N}$. We have $\mu_{X}\left(A_{n} \cap K_{p}\right)>\kappa-\left(\delta_{n}^{\prime}+\eta_{p}\right)$. Since $K_{p}$ is the compact set, $\left\{A_{n} \cap K_{p}\right\}_{m=1}^{\infty}$ has a Hausdorff convergent subsequence for any $p \in \mathbb{N}$. By a diagonal argument, we find a common subsequence $\{m(n)\}_{n=1}^{\infty} \subset \mathbb{N}$ such that $\left\{A_{m(n)} \cap K_{p}\right\}_{n=1}^{\infty}$ is a Hausdorff convergent sequence for any $p \in \mathbb{N}$. Denote its limit by $B_{p}$. For any $\varepsilon>0$, there exist $n_{0} \in \mathbb{N}$ such that $B_{p} \subset U_{\varepsilon}\left(A_{m(n)} \cap K_{p}\right)$ for any $n>n_{0}$. Since we can assume that $\delta_{m(n)}<\varepsilon$, we have $U_{r-2 \varepsilon}\left(B_{p}\right) \subset$ $U_{r-\delta_{m(n)}}\left(A_{m(n)} \cap K_{p}\right) .\left\{B_{p}\right\}_{p=1}^{\infty}$ is a monotone non-decreasing sequence of compact subsets of $X$ satisfying $\mu_{X}\left(B_{p}\right) \geq \kappa-\delta_{p}$ for any $p \in \mathbb{N}$. Setting

$$
B:=\bigcup_{p \in \mathbb{N}} B_{p}
$$

we have $\mu_{X}(B) \geq \kappa$ and $U_{r}(B)=\bigcup_{p \in \mathbb{N}} U_{r}\left(B_{p}\right)$. Since

$$
\beta \leq 1-\lim _{p \rightarrow \infty} \lim _{n \rightarrow \infty} \mu_{X}\left(U_{r-\delta_{m(n)}}\left(A_{m(n)} \cap K_{p}\right)\right)=1-\mu_{X}\left(U_{r}(B)\right),
$$

we obtain $\alpha_{X}(r, \kappa) \geq \beta$. This completes the proof.

Proposition 3.2 ([10, Proposition 1.2]). Let $X$ and $X^{\prime}$ be two mm-spaces. If $X^{\prime}$ is dominated by $X$, then we have

$$
\alpha_{X^{\prime}}(r, \kappa) \leq \alpha_{X}(r, \kappa)
$$

for any $r>0$ and $0<\kappa \leq 1$.

Definition 3.3 (Concentration function for a pyramid). Let $r>0$ and $0<\kappa \leq 1$. The $\kappa$-concentration function for a pyramid $\mathcal{P}$ is defined to be

$$
\alpha_{\mathcal{P}}(r, \kappa):=\lim _{\delta \rightarrow 0+} \sup _{X \in \mathcal{P}} \alpha_{X}(r-\delta, \kappa-\delta) .
$$

Proposition 3.4. For any $m m$-space $X$, we have

$$
\alpha_{\mathcal{P}_{X}}(r, \kappa)=\alpha_{X}(r, \kappa)
$$

for any $r>0$ and $0<\kappa \leq 1$.

Proof. The proposition follows from Proposition 3.2 and Lemma 3.1 
Lemma 3.5. Let $\mathcal{P}$ and $\mathcal{P}^{\prime}$ be two pyramids. If we have

$$
\mathcal{M}(\mathcal{P} ; 1, R) \subset U_{\varepsilon}\left(\mathcal{M}\left(\mathcal{P}^{\prime} ; 1, R\right)\right)
$$

for two positive real numbers $\varepsilon$ and $R$ with $2 \varepsilon<R$, then

$$
\alpha_{\mathcal{P}}(r, \kappa) \leq \alpha_{\mathcal{P}^{\prime}}(r-2 \varepsilon, \kappa-\varepsilon)+\varepsilon
$$

for any $2 \varepsilon<r<R$ and $0<\kappa \leq 1$.

Proof. We take any $\delta>0$ and any mm-space $X \in \mathcal{P}$. Let $a<\alpha_{X}(r-\delta, \kappa-\delta)$. There is a Borel subset $A$ such that $\mu_{X}(A) \geq \kappa-\delta$ and $a<1-\mu_{X}\left(U_{r-\delta}(A)\right)$. Define a 1-Lipschitz function $f: X \rightarrow[0, R]$ by $f(x):=\min \left\{d_{X}(x, A), R\right\}$ for $x \in X$. Then we have $f_{*} \mu_{X} \in \mathcal{M}(\mathcal{P} ; 1, R)$. By $\mathcal{M}(\mathcal{P} ; 1, R) \subset U_{\varepsilon}\left(\mathcal{M}\left(\mathcal{P}^{\prime} ; 1, R\right)\right)$, there are an mm-space $X^{\prime} \in \mathcal{P}^{\prime}$ and the 1-Lipschitz function $g: X^{\prime} \rightarrow[-R, R]$ such that $d_{\operatorname{Pr}}\left(f_{*} \mu_{X}, g_{*} \mu_{X^{\prime}}\right)<\varepsilon$. Let $B:=\left\{x^{\prime} \in X^{\prime} \mid g\left(x^{\prime}\right)<\varepsilon\right\}$. We see that

$$
\begin{aligned}
\mu_{X^{\prime}}(B) & =g_{*} \mu_{X^{\prime}}(\{t \in[-R, R] \mid t<\varepsilon\}) \\
& =g_{*} \mu_{X^{\prime}}\left(U_{\varepsilon}(\{t \in[-R, R] \mid t \leq 0\})\right) \\
& \geq f_{*} \mu_{X}(\{t \in[-R, R] \mid t \leq 0\})-\varepsilon \\
& =\mu_{X}(A)-\varepsilon \\
& \geq \kappa-(\delta+\varepsilon) .
\end{aligned}
$$

For any $x^{\prime} \in U_{r-(\delta+2 \varepsilon)}(B)$, there exists $y^{\prime} \in B$ such that $d_{X^{\prime}}\left(x^{\prime}, y^{\prime}\right)<r-(\delta+2 \varepsilon)$. The 1-Lipschitz continuity of $g$ implies that $g\left(x^{\prime}\right) \leq g\left(y^{\prime}\right)+r-(\delta+2 \varepsilon)<r-(\delta+\varepsilon)$. Then we have $U_{r-(\delta+2 \varepsilon)}(B) \subset B^{\prime}:=\left\{x^{\prime} \in X^{\prime} \mid g\left(x^{\prime}\right) \leq r-(\delta+\varepsilon)\right\}$. On the other hand, we see that

$$
\begin{aligned}
a & <1-\mu_{X}\left(U_{r-\delta}(A)\right) \\
& =1-f_{*} \mu_{X}(\{t \in[-R, R] \mid t<r-\delta\}) \\
& =1-f_{*} \mu_{X}\left(U_{\varepsilon}(\{t \in[-R, R] \mid t \leq r-(\delta+\varepsilon)\})\right) \\
& \leq 1-g_{*} \mu_{X^{\prime}}(\{t \in[-R, R] \mid t \leq r-(\delta+\varepsilon)\})+\varepsilon \\
& \leq 1-\mu_{X^{\prime}}\left(B^{\prime}\right)+\varepsilon \\
& \leq 1-\mu_{X^{\prime}}\left(U_{r-(\delta+2 \varepsilon)}(B)\right)+\varepsilon .
\end{aligned}
$$

This implies that $\alpha_{X}(r-\delta, \kappa-\delta) \leq \alpha_{X^{\prime}}(r-(\delta+2 \varepsilon), \kappa-(\delta+\varepsilon))+\varepsilon$. Taking supremums over $X^{\prime} \in \mathcal{P}^{\prime}, X \in \mathcal{P}$ and limit $\delta \rightarrow 0+$, we obtain

$$
\alpha_{\mathcal{P}}(r, \kappa) \leq \alpha_{\mathcal{P}^{\prime}}(r-2 \varepsilon, \kappa-\varepsilon)+\varepsilon .
$$

This completes the proof.

Corollary 3.6. Let $\mathcal{P}$ and $\mathcal{P}^{\prime}$ be two pyramids. If $\rho_{R}\left(\mathcal{P}, \mathcal{P}^{\prime}\right)<\varepsilon / 4$ for two positive real numbers $\varepsilon$ and $R$ with $2 \varepsilon<R$, then

$$
\alpha_{\mathcal{P}}(r, \kappa) \leq \alpha_{\mathcal{P}^{\prime}}(r-2 \varepsilon, \kappa-\varepsilon)+\varepsilon
$$

for any $2 \varepsilon<r<R$ and $0<\kappa \leq 1$.

Proof. Theorem 2.20 (4) implies that $\mathcal{M}(\mathcal{P} ; 1, R) \subset U_{\varepsilon}\left(\mathcal{M}\left(\mathcal{P}^{\prime} ; 1, R\right)\right)$. Using Lemma 3.5. we have the corollary. 
Proof of Theorem 1.1. For any real number $\delta>0$ with $r>4 \delta$ and $\kappa>2 \delta$, there is a number $n_{0}$ such that $\rho_{R}\left(\mathcal{P}_{n}, \mathcal{P}\right)<\delta / 4$ for any $n \geq n_{0}$. Let $n \geq n_{0}$. Corollary 3.6 implies

$$
\alpha_{\mathcal{P}}(r, \kappa)-\delta \leq \alpha_{\mathcal{P}_{n}}(r-2 \delta, \kappa-\delta) \leq \alpha_{\mathcal{P}}(r-4 \delta, \kappa-2 \delta)+\delta
$$

Taking the limits of this inequality as $n \rightarrow \infty$ and then $\delta \rightarrow 0+$, we obtain

$$
\begin{aligned}
\alpha_{\mathcal{P}}(r, \kappa) & =\lim _{\delta \rightarrow 0+} \liminf _{n \rightarrow \infty} \alpha_{\mathcal{P}_{n}}(r-\delta, \kappa-\delta) \\
& =\lim _{\delta \rightarrow 0+} \limsup _{n \rightarrow \infty} \alpha_{\mathcal{P}_{n}}(r-\delta, \kappa-\delta) .
\end{aligned}
$$

The proof is completed.

\section{Concentration FunCtion fOr A QMm-SPACE}

For a subset $A$ of a qmm-space $\left(Q, \mu_{Q}, d_{Q}^{*}\right)$ and for a real number $r>0$, we set

$$
U_{r}(A):=\left\{q \in Q \mid \uparrow d_{Q}^{*}(q, A)<r\right\},
$$

where $\uparrow d_{Q}^{*}(q, A):=\inf _{a \in A} \uparrow d_{Q}^{*}(q, a)$. Note that $A$ is not a subset of $U_{r}(A)$ in general.

Definition 4.1 (Concentration function for a qmm-space). Let $0<r, \kappa \leq 1$. The $\kappa$-concentration function for a qmm-space $Q$ is defined to be

$$
\alpha_{Q}(r, \kappa):=\lim _{\delta \rightarrow 0+} \sup \left\{1-\mu_{Q}\left(U_{r-\delta}(A)\right) \mid A \in \mathcal{B}(Q), \mu_{Q}(A) \geq \kappa-\delta\right\} .
$$

The next lemma is obvious from Theorem 2.28.

Lemma 4.2. Let $Q$ and $Q^{\prime}$ be two qmm-spaces. If $Q$ and $Q^{\prime}$ are isomorphic, then $\alpha_{Q}(r, \kappa)=\alpha_{Q^{\prime}}(r, \kappa)$ for any $0<r, \kappa \leq 1$.

Proposition 4.3. Let $X$ be an mm-space with diameter at most one. We have

$$
\alpha_{Q_{X}}(r, \kappa)=\alpha_{X}(r, \kappa)
$$

for any $0<r, \kappa \leq 1$.

Proof. The proposition follows from $\uparrow d_{Q_{X}}^{*}\left(x_{1}, x_{2}\right)=d_{X}\left(x_{1}, x_{2}\right)$ and Lemma 3.1

To prove Theorem 1.2, we recall ultraproducts of mm-spaces constructed in [3, Section 2.7] and [2, Section 3]. Let $\omega$ be a non-principal ultrafilter on $\mathbb{N}$, and $X_{n}, n=1,2, \ldots$, be mm-spaces with diameter at most one. For $\left\{x_{i}\right\}_{i=1}^{\infty},\left\{x_{i}^{\prime}\right\}_{i=1}^{\infty} \in$ $\prod_{n=1}^{\infty} X_{n},\left\{x_{i}\right\}_{i=1}^{\infty}$ and $\left\{x_{i}^{\prime}\right\}_{i=1}^{\infty}$ are equivalent if $\left\{i \in \mathbb{N} \mid x_{i}=x_{i}^{\prime}\right\} \in \omega$. Denote $\mathbf{X}$ by the set of equivalence classes. We can define a pseudo-metric on $\mathbf{X}$ by $\mathbf{d}_{\mathbf{X}}\left(\mathbf{x}, \mathbf{x}^{\prime}\right):=$ $\lim _{\omega} d_{X_{i}}\left(x_{i}, x_{i}^{\prime}\right)$, where $\left\{x_{i}\right\}_{i=1}^{\infty},\left\{x_{i}^{\prime}\right\}_{i=1}^{\infty}$ are representative elements of $\mathbf{x}, \mathbf{x}^{\prime} \in \mathbf{X}$, respectively. Let $A_{n}$ be Borel sets on $X_{n}, n=1,2, \ldots$ Define the ultraproduct set $\mathbf{A}$ in $\mathbf{X}$ in the following way: $\left[\left\{a_{i}\right\}\right] \in \mathbf{A}$ if and only if $\left\{i \in \mathbb{N} \mid a_{i} \in A_{i}\right\} \in \omega$. The set $\mathcal{U}$ of ultraproduct sets forms a Boolean algebra. Define the measure of ultraproduct set $\mathbf{A}$ by $\mu_{\mathbf{X}}(\mathbf{A}):=\lim _{\omega} \mu_{X_{i}}\left(A_{i}\right)$. Elek constructed a $\sigma$-algebra $\mathcal{S}$ containing $\mathcal{U}$ and extended the measure $\mu_{\mathbf{X}}$ on $\mathcal{S}$. We call $\left(\mathbf{X}, \mathbf{d}_{\mathbf{X}}, \mathcal{S}, \mu_{\mathbf{X}}\right)$ the ultraproduct of $\left\{X_{n}\right\}_{n=1}^{\infty}$.

Remark 4.4. (1) The function $\mathbf{d}_{\mathbf{X}}$ is not necessarily measurable on the product $\sigma$-algebra $\sigma(\mathcal{S} \times \mathcal{S})$. This is a measurable function on the ultraproduct $\left(\mathbf{X} \times \mathbf{X}, \mathbf{d}_{\mathbf{X}_{2}}, \mathcal{S}_{2}, \mu_{\mathbf{X}_{2}}\right)$ of $l_{\infty}$-products $\left\{X_{n} \times X_{n}\right\}_{n=1}^{\infty}$.

(2) The product $\sigma$-algebra $\sigma(\mathcal{S} \times \mathcal{S})$ is a sub- $\sigma$-algebra of $\mathcal{S}_{2}$. 
Lemma 4.5. Let $A_{n}$ be Borel sets on $X_{n}, n=1,2, \ldots$, and $\mathbf{A}$ its ultraproduct. Then we have $U_{r}(\mathbf{A}) \subset \lim _{\omega} U_{r}\left(A_{n}\right)$.

Proof. For any $\mathbf{x} \in U_{r}(\mathbf{A})$, there exist $\mathbf{x}^{\prime} \in \mathbf{A}$ such that $\mathbf{d}_{\mathbf{X}}\left(\mathbf{x}, \mathbf{x}^{\prime}\right)<r$. Denote $\left\{x_{i}\right\}_{i=1}^{\infty},\left\{x_{i}^{\prime}\right\}_{i=1}^{\infty}$ by representative elements of $\mathbf{x}, \mathbf{x}^{\prime} . \mathbf{d}_{\mathbf{X}}\left(\mathbf{x}, \mathbf{x}^{\prime}\right)<r$ implies that $\left\{i \in \mathbb{N} \mid d_{X_{i}}\left(x_{i}, x_{i}^{\prime}\right)<r\right\} \in \omega$. This means $\mathbf{x} \in \lim _{\omega} U_{r}(A)$.

Proposition 4.6 (Radon-Nikodym-Dundord-Pettis theorem, [2, Proposition 2.1]). Let $L$ be a Banach space, $L^{*}$ be its dual space with the weak topology, and $(\Omega, \mathcal{F}, \mathbb{P})$ a probability space. For any essentially bounded weak-*-measurable map $f: \Omega \rightarrow L^{*}$ and any sub- $\sigma$-algebra $\mathcal{F}^{\prime} \subset \mathcal{F}$, there exists an essentially unique map $E\left(f \mid \mathcal{F}^{\prime}\right)$ : $\Omega \rightarrow L^{*}$ which is weak-*-measurable with respect to $\mathcal{F}^{\prime}$ such that for any $v \in L$ and $A^{\prime} \in \mathcal{F}^{\prime}$ we have

$$
\int_{A^{\prime}}\left\langle E\left(f \mid \mathcal{F}^{\prime}\right)(x), v\right\rangle d \mathbb{P}(x)=\int_{A^{\prime}}\langle f(x), v\rangle d \mathbb{P}(x) .
$$

We call $E\left(f \mid \mathcal{F}^{\prime}\right)$ the Radon-Nikodym-Dundord-Pettis derivative of $f$. For the pseudo-metric $\mathbf{d}_{\mathbf{X}}$, we define the map $\delta_{\mathbf{d}_{\mathbf{X}}}: \mathbf{X} \times \mathbf{X} \rightarrow \mathcal{M}(I)$ by $\delta_{\mathbf{d}_{\mathbf{X}}}\left(\mathbf{x}, \mathbf{x}^{\prime}\right):=$ $\delta_{\mathbf{d}_{\mathbf{X}}\left(\mathbf{x}, \mathbf{x}^{\prime}\right)}$. Then $\delta_{\mathbf{d}_{\mathbf{X}}}$ is weak-*-measurable with respect to $\mathcal{S}_{2}$. Denote $\mathbf{d}_{\mathbf{X}}^{*}$ by the Radon-Nikodym-Dundord-Pettis derivative of $\delta_{\mathbf{d}_{\mathbf{x}}}$ with respect to $\sigma(\mathcal{S} \times \mathcal{S})$. Note that $\mathbf{d}_{\mathbf{X}}^{*}$ is an $\mathcal{M}(I)$-valued map.

Lemma 4.7. Let $\mathbf{A}$ be an ultraproduct set on $\left(\mathbf{X}, \mathbf{d}_{\mathbf{X}}, \mathcal{S}, \mu_{\mathbf{X}}\right)$ and $0<r<r^{\prime} \leq 1$. For $\mathbf{a} \in \mathbf{X}$, define the set $\mathbf{U}_{\mathbf{a}} \subset \mathbf{X}$ by

$$
\mathbf{U}_{\mathbf{a}}:=\left\{\mathbf{x} \in \mathbf{X} \mid \uparrow \mathbf{d}_{\mathbf{X}}^{*}(\mathbf{a}, \mathbf{x})<r, \mathbf{d}_{\mathbf{X}}(\mathbf{a}, \mathbf{x}) \geq r^{\prime}\right\} .
$$

Then we have $\mu_{\mathbf{X}}\left(\left\{\mathbf{a} \in \mathbf{A} \mid \mu_{\mathbf{X}}\left(\mathbf{U}_{\mathbf{a}}\right)>0\right\}\right)=0$.

Proof. It is trivial that $\mu_{\mathbf{X}}\left(\left\{\mathbf{a} \in \mathbf{A} \mid \mu_{\mathbf{X}}\left(\mathbf{U}_{\mathbf{a}}\right)>0\right\}\right)=0$ if $\mu_{\mathbf{X}}(\mathbf{A})=0$. We assume $\mu_{\mathbf{X}}(\mathbf{A})>0$. Define the set $\mathbf{U} \in \sigma(\mathcal{S} \times \mathcal{S})$ by

$$
\mathbf{U}:=\left\{(\mathbf{a}, \mathbf{x}) \in \mathbf{A} \times \mathbf{X} \mid \mathbf{x} \in \mathbf{U}_{\mathbf{a}}\right\} .
$$

$\mu_{\mathbf{X}}\left(\left\{\mathbf{a} \in \mathbf{A} \mid \mu_{\mathbf{X}}\left(\mathbf{U}_{\mathbf{a}}\right)>0\right\}\right)>0$ if and only if $\mu_{\mathbf{X}_{2}}(\mathbf{U})>0$. Let $g: I \rightarrow T$ be a continuous function satisfying $g(t)=1$ if $t \leq r$ and $g(t)=0$ if $t \geq\left(r+r^{\prime}\right) / 2$. Then if $\mu_{\mathbf{X}_{2}}(\mathbf{U})>0$,

$$
0<\int_{\mathbf{A}}\left\langle\mathbf{d}_{\mathbf{X}}^{*}\left(\mathbf{x}, \mathbf{x}^{\prime}\right), g\right\rangle d \mu_{\mathbf{X}}\left(\mathbf{x}, \mathbf{x}^{\prime}\right)=\int_{\mathbf{A}}\left\langle\delta_{\mathbf{d}_{\mathbf{X}}\left(\mathbf{x}, \mathbf{x}^{\prime}\right)}, g\right\rangle d \mu_{\mathbf{X}}\left(\mathbf{x}, \mathbf{x}^{\prime}\right)=0,
$$

leading to a contradiction. This completes the proof.

Theorem 4.8 ([2, Section 5-7]). Let $X_{n}, n=1,2, \ldots$, be $m m$-spaces and $Q$ be a $q m m$-space. If $X_{n}$ converges to $Q$ in sampling, then there exists an $\mathcal{S}$-measurable map $\mathbf{\Psi}: \mathbf{X} \rightarrow Q$ satisfying the following:

(1) $\boldsymbol{\Psi}(\mathcal{M}) \subset \mathcal{B}(Q)$.

(2) $\mu_{\mathbf{X}}(\mathbf{A})=\mu_{\mathbf{X}}\left(\boldsymbol{\Psi}^{-1}(\boldsymbol{\Psi}(\mathbf{A}))\right)$ for any ultraproduct set $\mathbf{A}$.

(3) $\mu_{Q}=\boldsymbol{\Psi}_{*} \mu_{\mathbf{X}}$.

(4) $\boldsymbol{\Psi}^{*} d_{Q}^{*}\left(\mathbf{x}, \mathbf{x}^{\prime}\right)=\mathbf{d}_{\mathbf{X}}^{*}\left(\mathbf{x}, \mathbf{x}^{\prime}\right)$ a.s. $\left(\mathbf{x}, \mathbf{x}^{\prime}\right) \in \mathbf{X} \times \mathbf{X}$.

Lemma 4.9. Let $Q$ be a qmm-space. For any sequence of mm-spaces $\left\{X_{n}\right\}_{n=1}^{\infty}$ such that $X_{n}$ converges to $Q$ in sampling, we have

$$
\lim _{\delta \rightarrow 0+} \limsup _{n \rightarrow \infty} \alpha_{X_{n}}(r-\delta, \kappa-\delta) \leq \alpha_{Q}(r, \kappa)
$$

for any $0<r, \kappa \leq 1$. 
Proof. Taking a subsequence of $\left\{\alpha_{X_{n}}(r-\delta, \kappa-\delta)\right\}_{n=1}^{\infty}$, we can suppose that the limit exists. There exists a Borel subset of $X_{n}$ with $\mu_{X_{n}}\left(A_{n}\right) \geq \kappa-\delta$ satisfying

$$
\alpha_{X_{n}}(r-\delta, \kappa-\delta)-\frac{1}{n} \leq 1-\mu_{X_{n}}\left(U_{r-\delta}\left(A_{n}\right)\right) .
$$

Denote $\mathbf{A}:=\lim _{\omega} A_{n}$. Then $\mu_{Q}(\mathbf{\Psi}(\mathbf{A}))=\mu_{\mathbf{X}}(\mathbf{A}) \geq \kappa-\delta$. Taking the ultralimit, by Lemma 4.5, Theorem 4.8 (2), (3), and Lemma 4.7, we have

$$
\begin{aligned}
\lim _{n \rightarrow \infty} \alpha_{X_{n}}(r-\delta, \kappa-\delta) & \leq 1-\mu_{\mathbf{X}}\left(\lim _{\omega} U_{r-\delta}\left(A_{n}\right)\right) \\
& \leq 1-\mu_{\mathbf{X}}\left(U_{r-\delta}(\mathbf{A})\right) \\
& =1-\mu_{\mathbf{X}}\left(\Psi^{-1}\left(\mathbf{\Psi}\left(U_{r-\delta}(\mathbf{A})\right)\right)\right) \\
& =1-\mu_{Q}\left(\mathbf{\Psi}\left(U_{r-\delta}(\mathbf{A})\right)\right) \\
& \leq 1-\mu_{Q}\left(U_{r-\delta / 2}(\mathbf{\Psi}(\mathbf{A}))\right) .
\end{aligned}
$$

By taking $\delta \rightarrow 0+$, we obtain the lemma.

Lemma 4.10. Let $Q$ be a qmm-space. There exists a sequence of mm-spaces $\left\{X_{n}\right\}_{n=1}^{\infty}$ such that $X_{n}$ converges to $Q$ in sampling such that

$$
\lim _{\delta \rightarrow 0+} \liminf _{n \rightarrow \infty} \alpha_{X_{n}}(r-\delta, \kappa-\delta) \geq \alpha_{Q}(r, \kappa)
$$

for any $0<r, \kappa \leq 1$. In particular, we have

$$
\begin{aligned}
\alpha_{Q}(r, \kappa) & =\lim _{\delta \rightarrow 0+} \liminf _{n \rightarrow \infty} \alpha_{X_{n}}(r-\delta, \kappa-\delta) \\
& =\lim _{\delta \rightarrow 0+} \limsup _{n \rightarrow \infty} \alpha_{X_{n}}(r-\delta, \kappa-\delta)
\end{aligned}
$$

for any $0<r, \kappa \leq 1$.

Proof. We recall random mm-spaces $\left\{X_{n}\right\}_{n=1}^{\infty}$ constructed by Elek [2, Section 6]. Let us pick a sequence $\left\{x_{k}\right\}_{k=1}^{\infty} \in Q^{\infty}$ of independent $\mu_{Q}^{\otimes \infty}$-random points in $Q$. For each pair $\left(x_{i}, x_{j}\right)$, we pick $t_{i, j} \in I$ independently according to $d_{Q}^{*}\left(x_{i}, x_{j}\right)$. Denote the mm-space $X_{n}$ by $X_{n}=\left\{x_{k}\right\}_{k=1}^{n}, d_{X_{n}}\left(x_{i}, x_{j}\right)=t_{i, j}$, and $\mu_{X_{n}}=n^{-1} \sum_{k=1}^{n} \delta_{x_{k}}$. Then $\left\{X_{n}\right\}_{n=1}^{\infty}$ converges to $Q$ in sampling and with probability one.

Let $\delta>0$. There exists $A_{\delta} \in \mathcal{B}(Q)$ such that $\mu_{Q}\left(A_{\delta}\right) \geq \kappa-\delta$ and

$$
\alpha_{Q}(r, \kappa)=1-\lim _{\delta \rightarrow 0+} \mu_{Q}\left(U_{r-\delta}\left(A_{\delta}\right)\right) .
$$

Denote the set $B_{n, \delta}:=X_{n} \cap A_{\delta}$. By the definition of $\mu_{X_{n}}$, we have $\mu_{X_{n}}\left(B_{n, \delta}\right)=$ $\mu_{X_{n}}\left(A_{\delta}\right)$ and $\mu_{X_{n}}\left(U_{r-\delta}\left(B_{n, \delta}\right)\right) \leq \mu_{X_{n}}\left(U_{r-\delta}\left(A_{\delta}\right)\right)$. Since $\left\{X_{n}\right\}_{n=1}^{\infty}$ converges to $Q$ in sampling, we have

$$
\lim _{n \rightarrow \infty} \mu_{X_{n}}\left(B_{n, \delta}\right)=\mu_{Q}\left(A_{\delta}\right) \geq \kappa-\delta
$$

and

$$
\limsup _{n \rightarrow \infty} \mu_{X_{n}}\left(U_{r-\delta}\left(B_{n, \delta}\right)\right) \leq \mu_{Q}\left(U_{r-\delta}\left(A_{\delta}\right)\right) .
$$

This implies that

$$
\lim _{\delta \rightarrow 0+} \liminf _{n \rightarrow \infty} \alpha_{X_{n}}(r-\delta, \kappa-\delta) \geq \alpha_{Q}(r, \kappa) .
$$

We obtain the lemma. 
Proof of Theorem 1.2. For any $Q_{n}$, there exist a sequence of mm-spaces $\left\{X_{n, m}\right\}_{m=1}^{\infty}$ as in Lemma 4.10. Then $X_{n, n}$ converges to $Q$ in sampling. Combining Lemma 4.9 and Lemma 4.10, we have

$$
\lim _{\delta \rightarrow 0+} \limsup _{n \rightarrow \infty} \alpha_{Q_{n}}(r-\delta, \kappa-\delta) \leq \lim _{\delta \rightarrow 0+} \limsup _{n \rightarrow \infty} \alpha_{X_{n, n}}(r-\delta, \kappa-\delta) \leq \alpha_{Q}(r, \kappa) .
$$

This completes the proof.

Remark 4.11. If $Q$ and $Q_{n}, n=1,2, \ldots$, are mm-spaces, the $\square$-convergence implies

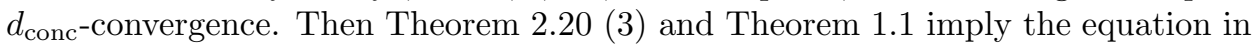
Theorem 1.2

Denote $d_{H}^{*}: I^{2} \rightarrow \mathcal{M}(I)$ by $d_{H}^{*}(t, t):=\delta_{0}$ and $d_{H}^{*}\left(t, t^{\prime}\right):=\delta_{1 / 2}$ if $t \neq t^{\prime}$.

Proposition 4.12. $\left\{S^{n}\left(\pi^{-1}\right)\right\}_{n=1}^{\infty}$ converges to $H=\left(I, \mathcal{L}, d_{H}^{*}\right)$ in sampling as $n \rightarrow \infty$.

Proof. For $x_{n}^{\prime} \in S^{n}\left(\pi^{-1}\right)$, denote its distance function by $f_{n}\left(x_{n}\right):=d_{S^{n}\left(\pi^{-1}\right)}\left(x_{n}, x_{n}^{\prime}\right)$. Then Lévy's lemma implies that

$$
\lim _{n \rightarrow \infty} d_{\mathrm{KF}}^{\mu_{S^{n}\left(\pi^{-1}\right)}}\left(f_{n}, 2^{-1}\right)=0 .
$$

In particular we have

$$
\lim _{n \rightarrow \infty} d_{\mathrm{KF}}^{\mu_{S^{n}\left(\pi^{-1}\right)}^{\otimes 2}}\left(d_{S^{n}\left(\pi^{-1}\right)}, 2^{-1}\right)=0 .
$$

Denote $H_{N}=\left(h_{i, j}\right)_{i, j=1}^{N}$ by the element of $M_{N}(I)$ satisfying that diagonal elements are zero and off-diagonal elements are $1 / 2$. Define the map $F_{N}:\left(S^{n}\left(\pi^{-1}\right)\right)^{N} \rightarrow M_{N}(I)$ by $F_{N}\left(x_{1}, \ldots, x_{N}\right):=H_{N}$. Let $\left\{\varepsilon_{n}\right\}_{n=1}^{\infty}$ be a monotone decreasing sequence of positive real numbers converging to zero with $d_{\mathrm{KF}}^{\mu_{S^{n}\left(\pi^{-1}\right)}^{\otimes 2}}\left(d_{S^{n}\left(\pi^{-1}\right)}, 2^{-1}\right)<\varepsilon_{n}$. Define the set $V_{N} \subset X^{N}$ by

$$
\begin{gathered}
V_{N}:=\left\{\left(x_{1}, \ldots, x_{N}\right) \in X^{N}|| d_{S^{n}\left(\pi^{-1}\right)}\left(x_{i}, x_{j}\right)-h_{i, j} \mid \leq \varepsilon_{n}\right. \\
\text { for all } i, j=1, \ldots, N\} .
\end{gathered}
$$

Then we have $\mu_{S^{n}\left(\pi^{-1}\right)}^{\otimes N}\left(V_{N}\right)>1-N^{2} \varepsilon_{n}$. We prove

$$
F_{N}^{-1}(A) \cap V_{N} \subset\left(K_{N}^{S^{n}\left(\pi^{-1}\right)}\right)^{-1}\left(U_{N^{2} \varepsilon_{n}}(A)\right)
$$

for any $A \in \mathcal{B}\left(M_{N}(I)\right)$. For any $x \in F_{N}^{-1}(A) \cap V_{N}, F_{N}(x) \in A$ and $x \in V_{N}$, which imply $K_{N}^{S^{n}\left(\pi^{-1}\right)}(x) \in U_{N^{2} \varepsilon_{n}}(A)$ and so $x \in\left(K_{N}^{S^{n}\left(\pi^{-1}\right)}\right)^{-1}\left(U_{N^{2} \varepsilon_{n}}(A)\right)$. Thus, we have $F_{N}^{-1}(A) \cap V_{N} \subset\left(K_{N}^{S^{n}\left(\pi^{-1}\right)}\right)^{-1}\left(U_{N^{2} \varepsilon_{n}}(A)\right)$. Since

$$
\mu_{S^{n}\left(\pi^{-1}\right)}^{\otimes N}\left(F_{N}^{-1}(A) \backslash V_{N}\right) \leq \mu_{S^{n}\left(\pi^{-1}\right)}^{\otimes N}\left(\left(S^{n}\left(\pi^{-1}\right)\right)^{N} \backslash V_{N}\right) \leq N^{2} \varepsilon_{n},
$$

we have

$$
\begin{aligned}
\delta_{H_{N}}(A) & =\left(F_{N}\right)_{*} \mu_{S^{n}\left(\pi^{-1}\right)}^{\otimes N}(A) \\
& =\mu_{S^{n}\left(\pi^{-1}\right)}^{\otimes N}\left(F_{N}^{-1}(A)\right) \\
& =\mu_{S^{n}\left(\pi^{-1}\right)}^{\otimes N}\left(F_{N}^{-1}(A) \cap V_{N}\right)+\mu_{S^{n}\left(\pi^{-1}\right)}^{\otimes N}\left(F_{N}^{-1}(A) \backslash V_{N}\right) \\
& \leq \mu_{S^{n}\left(\pi^{-1}\right)}^{\otimes N}\left(\left(K_{N}^{S^{n}\left(\pi^{-1}\right)}\right)^{-1}\left(U_{N^{2} \varepsilon_{n}}(A)\right)\right)+N^{2} \varepsilon_{n} \\
& =\underline{\mu}_{N}^{S^{n}\left(\pi^{-1}\right)}\left(U_{N^{2} \varepsilon_{n}}(A)\right)+N^{2} \varepsilon_{n} .
\end{aligned}
$$


This implies that $d_{\operatorname{Pr}}\left(\underline{\mu}_{N}^{S^{n}\left(\pi^{-1}\right)}, \delta_{H_{N}}\right) \leq N^{2} \varepsilon_{n}$; then $\underline{\mu}_{N}^{S^{n}\left(\pi^{-1}\right)}$ converses weakly to $\delta_{H_{N}}$ as $n \rightarrow \infty$ for any $N \in \mathbb{N}$. By Lemma 2.6. $\underline{\mu}_{\infty}^{S^{n}\left(\pi^{-1}\right)}$ converges weakly to $\delta_{H_{\infty}}$ as $n \rightarrow \infty$. Since the representative qmm-space of $\delta_{H_{\infty}}$ is $H$, we obtain the proposition.

Remark 4.13. Lemma 2.8 and the well-known estimation in [8, Section 1]

$$
\alpha_{S^{n}\left(\pi^{-1}\right)}(r, 1 / 2) \leq \sqrt{2} \exp \left(-\pi(n-1) r^{2} / 2\right)
$$

implies

$$
\lim _{\delta \rightarrow 0+} \limsup _{n \rightarrow \infty} \alpha_{S^{n}\left(\pi^{-1}\right)}(r-\delta, 1 / 2-\delta)=0
$$

for any $r>0$ but $\alpha_{H}(r, 1 / 2)=1 / 2$ for any $0<r \leq 1 / 2$. By the above computation, we can see that

$$
\alpha_{Q}(r, \kappa) \leq \lim _{\delta \rightarrow 0+} \liminf _{n \rightarrow \infty} \alpha_{Q_{n}}(r-\delta, \kappa-\delta)
$$

does not hold in general.

\section{ACKNOWLEDGEMENT}

The author would like to thank Professor Takashi Shioya for valuable discussions and many fruitful suggestions.

\section{REFERENCES}

[1] D. Amir and V. D. Milman, Unconditional and symmetric sets in $n$-dimensional normed spaces, Israel J. Math. 37 (1980), no. 1-2, 3-20, DOI 10.1007/BF02762864. MR.599298

[2] G. Elek, Samplings and observables. Invariants of metric measure spaces, arXiv:1205.6936, preprint.

[3] Gábor Elek and Balázs Szegedy, A measure-theoretic approach to the theory of dense hypergraphs, Adv. Math. 231 (2012), no. 3-4, 1731-1772, DOI 10.1016/j.aim.2012.06.022. MR.2964622

[4] Kei Funano, Estimates of Gromov's box distance, Proc. Amer. Math. Soc. 136 (2008), no. 8, 2911-2920, DOI 10.1090/S0002-9939-08-09416-1. MR2399058

[5] K. Funano, Asymptotic behavior of mm-spaces, Doctoral Thesis, Tohoku University (2009).

[6] Andreas Greven, Peter Pfaffelhuber, and Anita Winter, Convergence in distribution of random metric measure spaces ( $\Lambda$-coalescent measure trees), Probab. Theory Related Fields 145 (2009), no. 1-2, 285-322, DOI 10.1007/s00440-008-0169-3. MR2520129

[7] Misha Gromov, Metric structures for Riemannian and non-Riemannian spaces, Reprint of the 2001 English edition, Modern Birkhäuser Classics, Birkhäuser Boston, Inc., Boston, MA, 2007. Based on the 1981 French original; With appendices by M. Katz, P. Pansu and S. Semmes; Translated from the French by Sean Michael Bates. MR2307192

[8] M. Gromov and V. D. Milman, A topological application of the isoperimetric inequality, Amer. J. Math. 105 (1983), no. 4, 843-854, DOI 10.2307/2374298. MR708367

[9] Takefumi Kondo, Probability distribution of metric measure spaces, Differential Geom. Appl. 22 (2005), no. 2, 121-130, DOI 10.1016/j.difgeo.2004.10.001. MR2122737

[10] Michel Ledoux, The concentration of measure phenomenon, Mathematical Surveys and Monographs, vol. 89, American Mathematical Society, Providence, RI, 2001. MR.1849347

[11] Wolfgang Löhr, Equivalence of Gromov-Prohorov- and Gromov's $\square_{\lambda}$-metric on the space of metric measure spaces, Electron. Commun. Probab. 18 (2013), no. 17, 10. MR.3037215

[12] László Lovász and Balázs Szegedy, Limits of dense graph sequences, J. Combin. Theory Ser. B 96 (2006), no. 6, 933-957, DOI 10.1016/j.jctb.2006.05.002. MR.2274085

[13] Ryunosuke Ozawa and Takashi Shioya, Limit formulas for metric measure invariants and phase transition property, Math. Z. 280 (2015), no. 3-4, 759-782, DOI 10.1007/s00209-0151447-2. MR3369350

[14] Ryunosuke Ozawa and Takashi Shioya, Estimate of observable diameter of $l_{p}$-product spaces, Manuscripta Math. 147 (2015), no. 3-4, 501-509, DOI 10.1007/s00229-015-0730-1. MR.3360754 
[15] Takashi Shioya, Metric measure geometry, IRMA Lectures in Mathematics and Theoretical Physics, vol. 25, EMS Publishing House, Zürich, 2016. Gromov's theory of convergence and concentration of metrics and measures. MR 3445278

[16] T. Shioya, Metric measure limit of spheres and complex projective spaces, arXiv:1402.0611, preprint

[17] A. M. Vershik, The universal Uryson space, Gromov's metric triples, and random metrics on the series of natural numbers (Russian), Uspekhi Mat. Nauk 53 (1998), no. 5(323), 5764, DOI 10.1070/rm1998v053n05ABEH000069; English transl., Russian Math. Surveys 53 (1998), no. 5, 921-928. MR.1691182

Max Planck Institute for Mathematics, Vivatsgasse 7, 53111 Bonn, Germany

E-mail address: ozawa@mpim-bonn.mpg.de 